\title{
Identification of the segmental structures of the right upper lobe of the lung using non-enhanced thin-slice CT
}

\author{
Renfeng Wang ${ }^{1 \#}$, Yi Zhang ${ }^{2 \#}$, Quanteng $\mathrm{Hu}^{3}, \mathrm{Ke} \mathrm{Jin}^{3}$, Gang Huang ${ }^{4}$, Jianfei Shen $^{3 *}$, Feichao Bao ${ }^{5 *}$ \\ ${ }^{1}$ Department of Thoracic Surgery, First Hospital of Shijiazhuang, Shijiazhuang 050000, China; ${ }^{2}$ Department of Thoracic Surgery, Zhongshan \\ Hospital, Fudan University, Shanghai 200000, China; ${ }^{3}$ Department of Cardiothoracic Surgery, Taizhou Hospital of Zhejiang Province, Wenzhou \\ Medical University, Linhai 317000, China; ${ }^{4}$ Department of Thoracic Surgery, The Third Hospital of Hebei Medical University, Shijiazhuang \\ 050000, China; ${ }^{5}$ Department of Thoracic Surgery, Shanghai Chest Hospital, Shanghai Jiao Tong University, Shanghai 200000, China \\ \#These authors contributed equally to this work. \\ *Senior authors contributed equally to this work. \\ Correspondence to: Mr. Jianfei Shen. Department of Cardiothoracic Surgery, Taizhou Hospital, Wenzhou Medical University, Linhai 317000 , China. \\ Email: shenjf9717@enzemed.com; Mr. Feichao Bao. Department of Thoracic Surgery, Shanghai Chest Hospital, Shanghai Jiao Tong University, \\ Shanghai 200000, China. Email: baofeichao@126.com. \\ Provenance and Peer Review: This article was commissioned by the Editorial Office, fournal of Thoracic Disease. The article did not undergo external \\ peer review.
}

Submitted Jan 27, 2020. Accepted for publication Feb 26, 2020.

doi: $10.21037 /$ jtd.2020.03.56

View this article at: http://dx.doi.org/10.21037/jtd.2020.03.56

\section{Introduction}

Lung cancer, with approximately 1.8 million new cases globally each year and over 1.6 million related deaths, remains the leading cause of cancer mortality (1). In recent years, the spectrum of lung cancer has changed drastically with the increasing application of computed tomography (CT)-based screening. Advanced localized central lung squamous cell carcinoma was once the dominant histologic type; however, nowadays, early lung adenocarcinoma radiologically featured by isolated pulmonary nodule, ground glass nodules (GGNs), and sub-centimeter-sized nodules is more common (2).

In 1995, a randomized controlled trial reported by the Lung Cancer Study Group (LCSG) found that lobectomy achieved a superior impact on the prognosis of patients with peripheral early non-small cell lung cancer (NSCLC) compared with limited pulmonary resection (3). However, with the change in the disease spectrum, further investigation is warranted to determine whether traditional lobectomy remains the optimal treatment for early stage lung cancer. With the recent major breakthroughs in prevention and early detection, molecular diagnostics, surgery, adjuvant therapy, radiotherapy, molecular targeted therapy, and immunotherapy, the treatment of lung cancer has increasingly been standardized and individualized, with regard to patients with early stage lung cancer, especially to those with limited pulmonary function, the main concern was to preserve more lung function without compromising oncologic effect. Segmentectomy would be an optional treatment to preserve more lung function when compared with lobectomy.

Several retrospective studies have shown pulmonary segmentectomy to be equally effective as lobectomy, and no significant difference has been shown in the overall survival and recurrence-free survival rates between the treatments. Meanwhile, pulmonary segmentectomy can better preserve postoperative pulmonary function (4). Thus, as a more minimally invasive technique, the role of pulmonary segmentectomy in treating early-stage lung cancer has increasingly been recognized among thoracic surgeons.

Sublobar resection for lung cancer includes two distinct procedures: non-anatomical resection (wedge resection) and anatomical resection (pulmonary segmentectomy). The difference between these two procedures is that pulmonary segmentectomy requires the surgeon to adhere to the oncological principles of lobectomy, which include the anatomical dissection of vein/arteries and bronchus in pulmonary segments and appropriate removal of the target lung parenchymal tissue. Compared with wedge resection, 

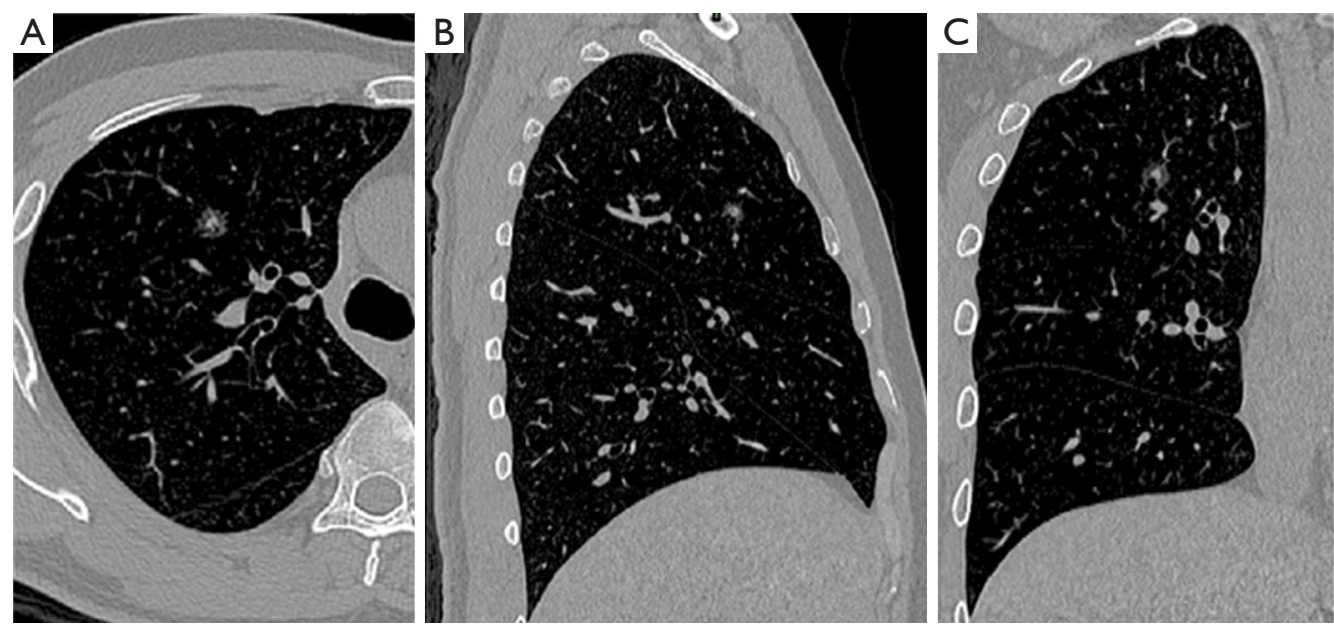

Figure 1 Location of the nodule on CT (C/T value: 0.399). (A) Transverse position; (B) coronal position; (C) sagittal position. CT, computed tomography.

segmentectomy can maintain a sufficient surgical margin from the tumor, which helps to maintain the morphology of residual lungs. Dissection of lymph nodes among the lung segments can reduce local recurrence and metastasis of malignant tumors, which has also led to the procedure becoming more popular for both surgeons and patients. However, pulmonary segmentectomy is a complicated and technical demanding procedure. Surgeons must undergo rigorous training to learn this procedure and to have a good grasp of its standardized steps. And, of these steps, preoperative planning is particularly important.

Reading CT images is a basic skill for thoracic surgeons. Based on CT images, the anatomic structures (including trachea, arteries, and veins) of the targeted segment and neighboring segment must be precisely identified before operation to ensure the operation proceeded smoothly. In this article, we described the process to identify anatomic segmental structures in the right upper lobe by using thinslice CT scans of a patient with lung cancer in his right upper lobe.

\section{Case presentation}

A 50-year-old man was found a $1.0 \mathrm{~cm}$-sized mixed ground glass nodule (GGN) in the anterior segment of the right upper lung (Figure 1). After one year of follow-up, the solid component of the nodule increased. Because the nodule was close to the V1b plane which was the border between the apical segment and anterior segment, and the anterior segmental bronchus (B3) was originated from
$\mathrm{S} 1 \mathrm{~b}$, combined segmentectomy, $\mathrm{RS} 3+\mathrm{S} 1 \mathrm{~b}$ resection was performed for this patient.

With this patient as an example, six typical layers from the caudal part to cranial part are labeled to demonstrate the key anatomic structures of right upper lobe (Figure 2). The Bronchus is of four-branch type, with $\mathrm{B} 1 \mathrm{a}$ originates from $\mathrm{B} 2$ and $\mathrm{B} 1 \mathrm{~b}$ from $\mathrm{B} 3$. For segmental arteries, $\mathrm{A} 3 \mathrm{a}$ is the anterior ascending branch, $\mathrm{A} 3 \mathrm{~b}+\mathrm{A} 1$ is the apical and anterior branch, the two above originate from the right pulmonary artery trunk separately, $\mathrm{A} 2$ is a posterior ascending branch, there is no recurrent $\mathrm{A} 2$. The vein is of $\mathrm{V} 1 \mathrm{~b}+$ central vein type, vein of S1b which needs to be ligated during RS3 + S1b resection, was drain into right upper vein separately with the central vein (Figure 3).

The concept of the vein mesh plane: The introduction of the "vein mesh plane" has confirmed that the vein plane is a curved or staggered surface rather than a simple single plane. In this case, V2c and V2b had two spatially arranged upper and lower branches, which also formed the corresponding spatial planes.

The V1b mesh plane is the border between the anterior segment and the apical segment (composed of veins 1, 2, 3, 4, 5, and 6), the V2a mesh plane is the border between the apical segment and the posterior segment (composed of veins $6,5,7,8,9,10,11$, and 12 ), and the V1a mesh plane is the border between S1a and S1b (composed of veins 6, 5, 7, 13, 14, and 15) (Figure 4).

Each vein mesh plane represents one segment or subsegment border, which divides the segment from the sub-segment. Theoretically, when electrocoagulation is 

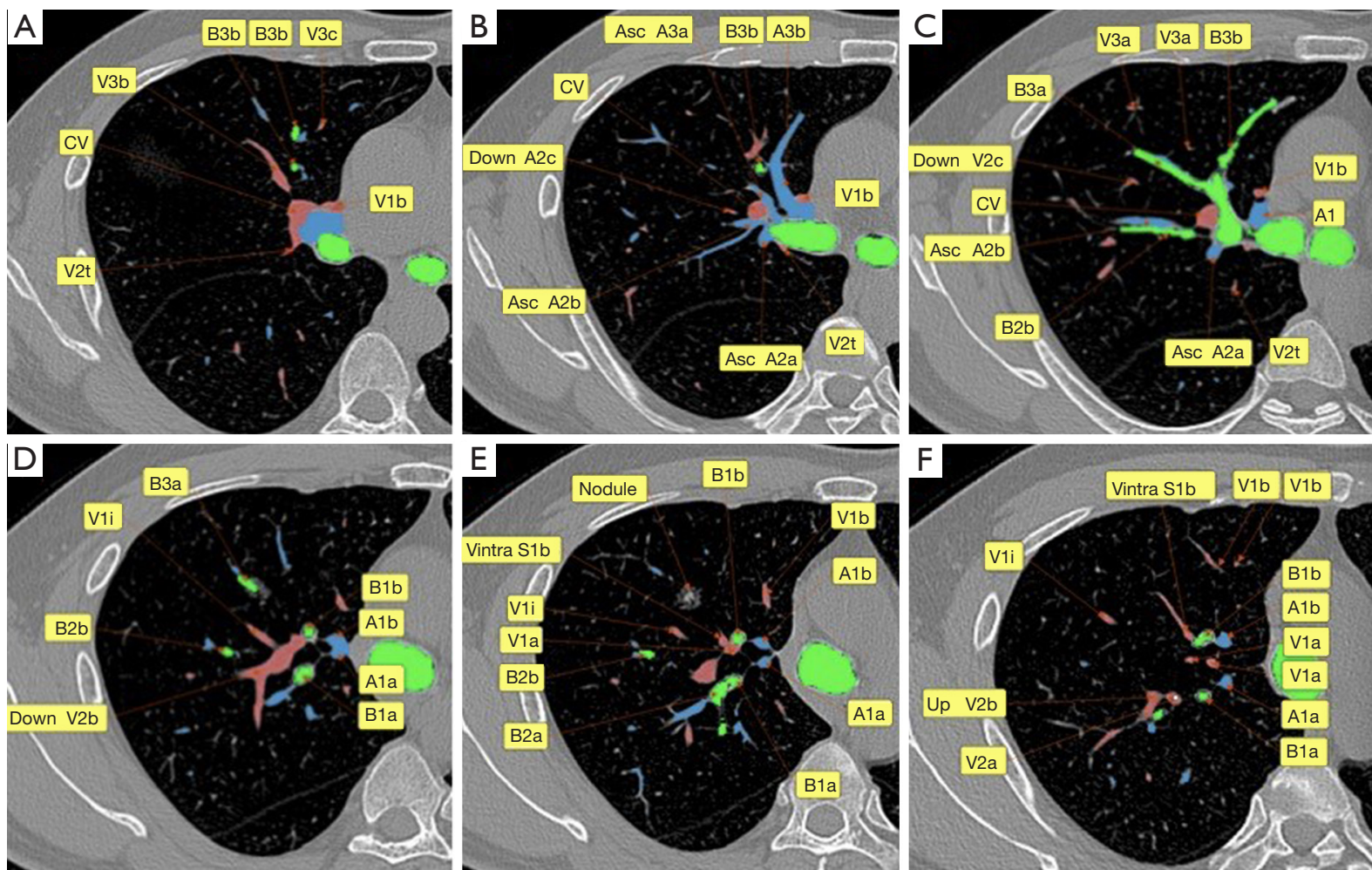

Figure 2 Anatomical features displayed in six typical layers of trachea, arteries, and veins. (A) The plane on which V3b is located; (B) the plane on which both Asc A2 and Asc A3a are displayed; (C) the plane on which both B3 and B2b are displayed; (D) the plane on which S1 artery/vein and V1i are located; (E) the plane on which the nodule is located; (F) typical V1a.
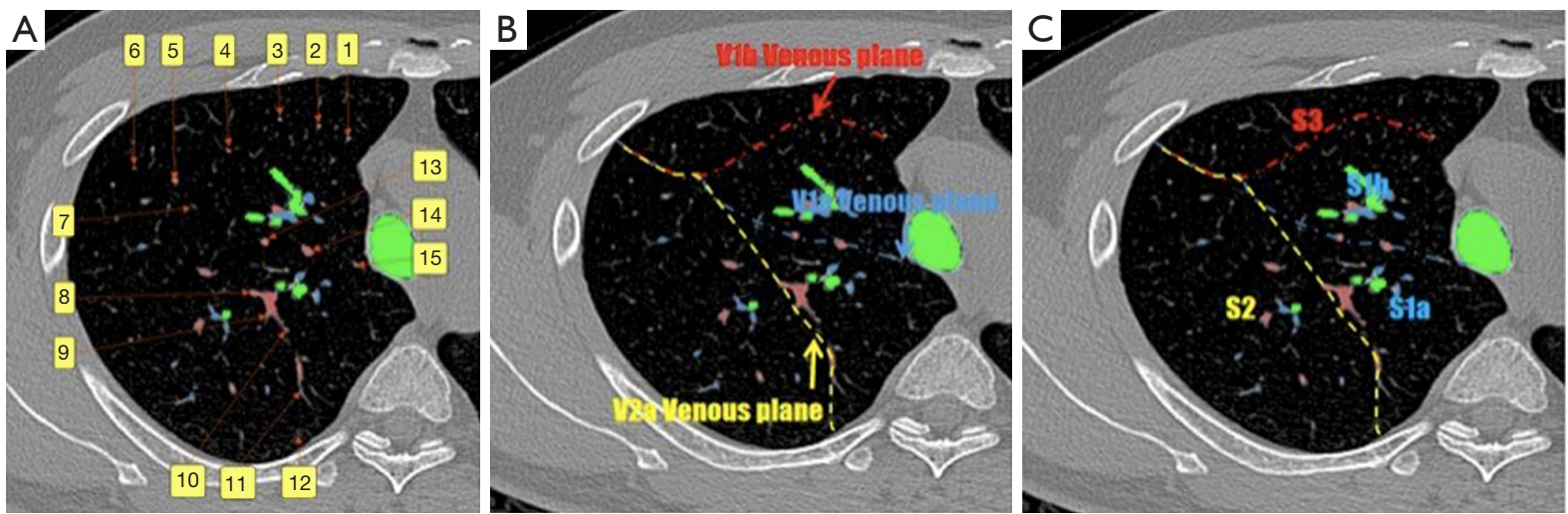

Figure 3 Concept of vein mesh plane. (A) Digital identification of distal multiple veins; (B) display of V1a, V1b, and V2a vein mesh; (C) display of the segments and subsegments in the upper right lung after the division of vein network. 
A
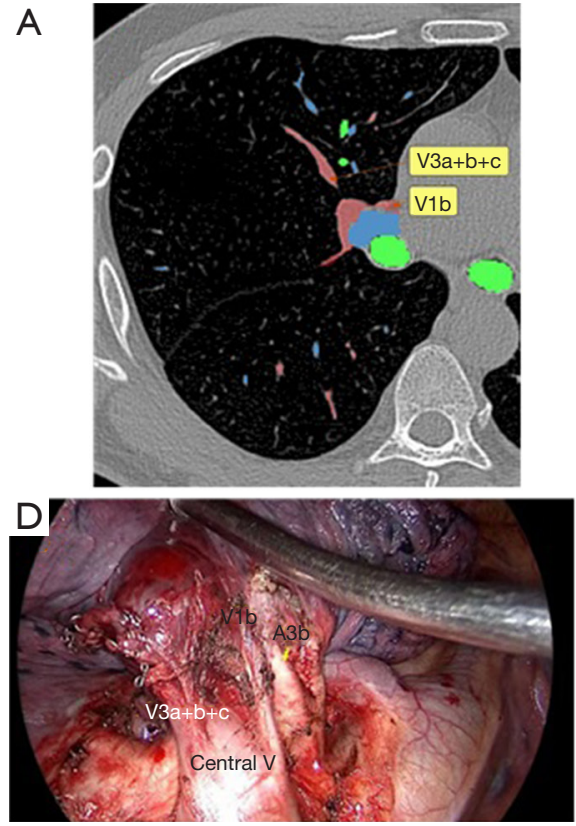

B
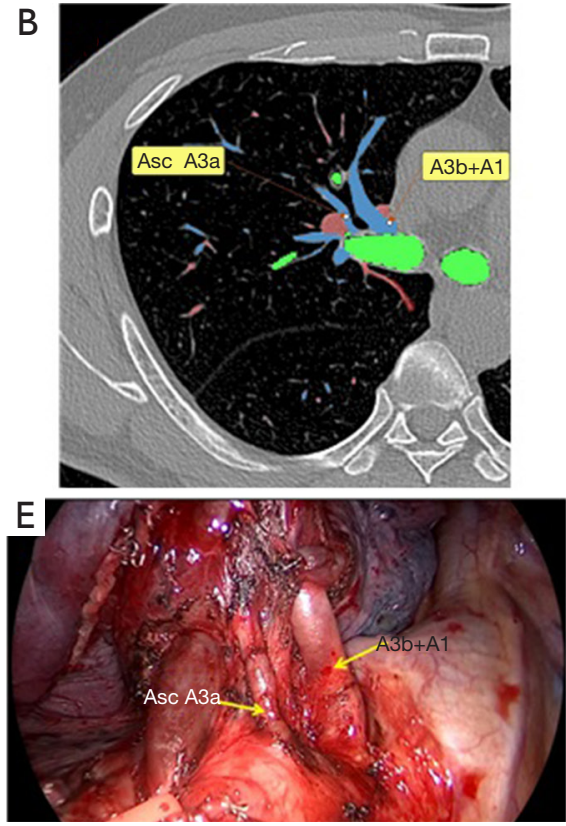

C
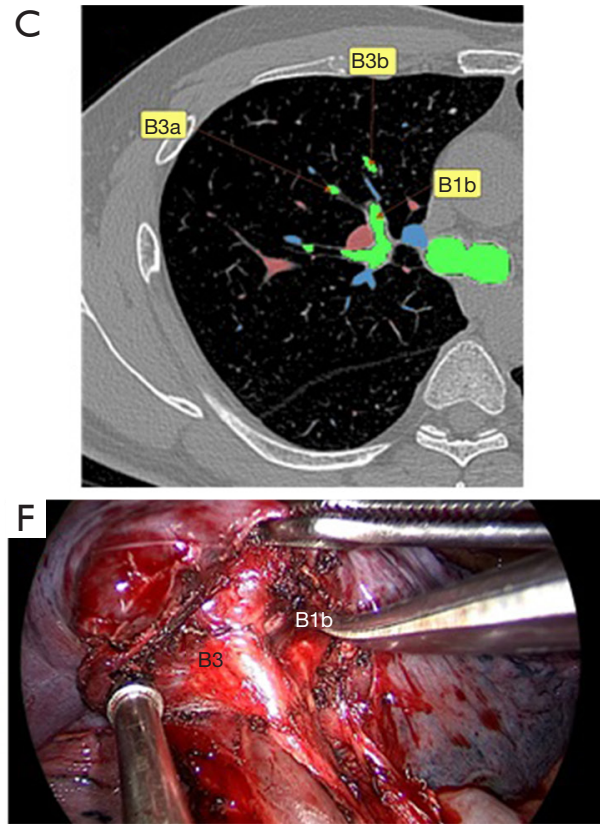

Figure 4 Comparison of 2D CT findings with intraoperative findings. (A,D) V1b and V3a+b+c; (B,E) Asc A3a and A3b+A1; (C,F) B3 and B1b.

applied to split the intersegmental plane, the veins forming the mesh should be visible as they are located on the intersegmental plane.

Comparison of intraoperative conditions with twodimensional CT findings: the planned surgical path would be of the following: $\mathrm{V} 3 \mathrm{a}+\mathrm{b}+\mathrm{c} \rightarrow \mathrm{V} 1 \mathrm{~b} \rightarrow$ Asc A3a $\rightarrow \mathrm{A} 3 \mathrm{~b}$ $\rightarrow \mathrm{A} 1 \mathrm{~b} \rightarrow \mathrm{B} 3+\mathrm{B} 1 \mathrm{~b} \rightarrow \operatorname{VintraS1b}$ (Figure 5 ).

\section{Discussion}

Compared with traditional lobectomy, segmentectomy can maximize lung function preservation and thus is a feasible treatment for a variety of benign and malignant diseases. Concerning malignant diseases, segmentectomy is mainly appropriate for treating patients with earlystage lung cancer. A retrospective study has shown how segmentectomy and lobectomy can achieve the same level of effectiveness in treating early-stage small lung cancer (4). According to the NCCN guidelines, segmentectomy is suitable for patients with compromised pulmonary function who cannot tolerate a lobectomy or for patients with small lung cancers $(<2.0 \mathrm{~cm})$ who meet any of the following criteria: (I) the pathological type is AIS; (II) the GGO component $>50 \%$; or (III) imaging monitoring suggested that the doubling time was $>400 \mathrm{~d}$. If segmentectomy is to be used as a standard treatment for early-stage lung cancer, many problems other than its oncological efficacy (i.e., survival) must be addressed. Segmentectomy has its own problems including the accurate identification of the intersegmental plane, resection margin, lymph node metastasis outside the target segment, anatomical malformation, and control of postoperative complications. The optimal indications of segmentectomy are still being investigated in two randomized controlled trials (CALGB 140503 and JCOG0802) (5).

Understanding the segmental anatomy would be helpful to recognize the anatomical variations frequently occurred in pulmonary segmentectomy. Preoperative three-dimensional (3D) CT reconstruction can identify the anatomical structures of bronchi, arteries, and veins in the lung segments and the relationships between the lesion and the surrounding structures. 3D CT reconstruction requires special thinslice 2D CT findings as the original data as well as certain software and technical supports. Different reconstruction techniques have different results. It would bring mislead for inexperienced surgeons, and should be corrected through the combination of $2 \mathrm{D} \mathrm{CT}$ and $3 \mathrm{D}$ reconstruction. As the first-hand information source, thin-slice CT can avoid data loss. Our current article describes a method for learning how to identify segmental structures based on 2D CT, although this method also requires long-term training and learning. 

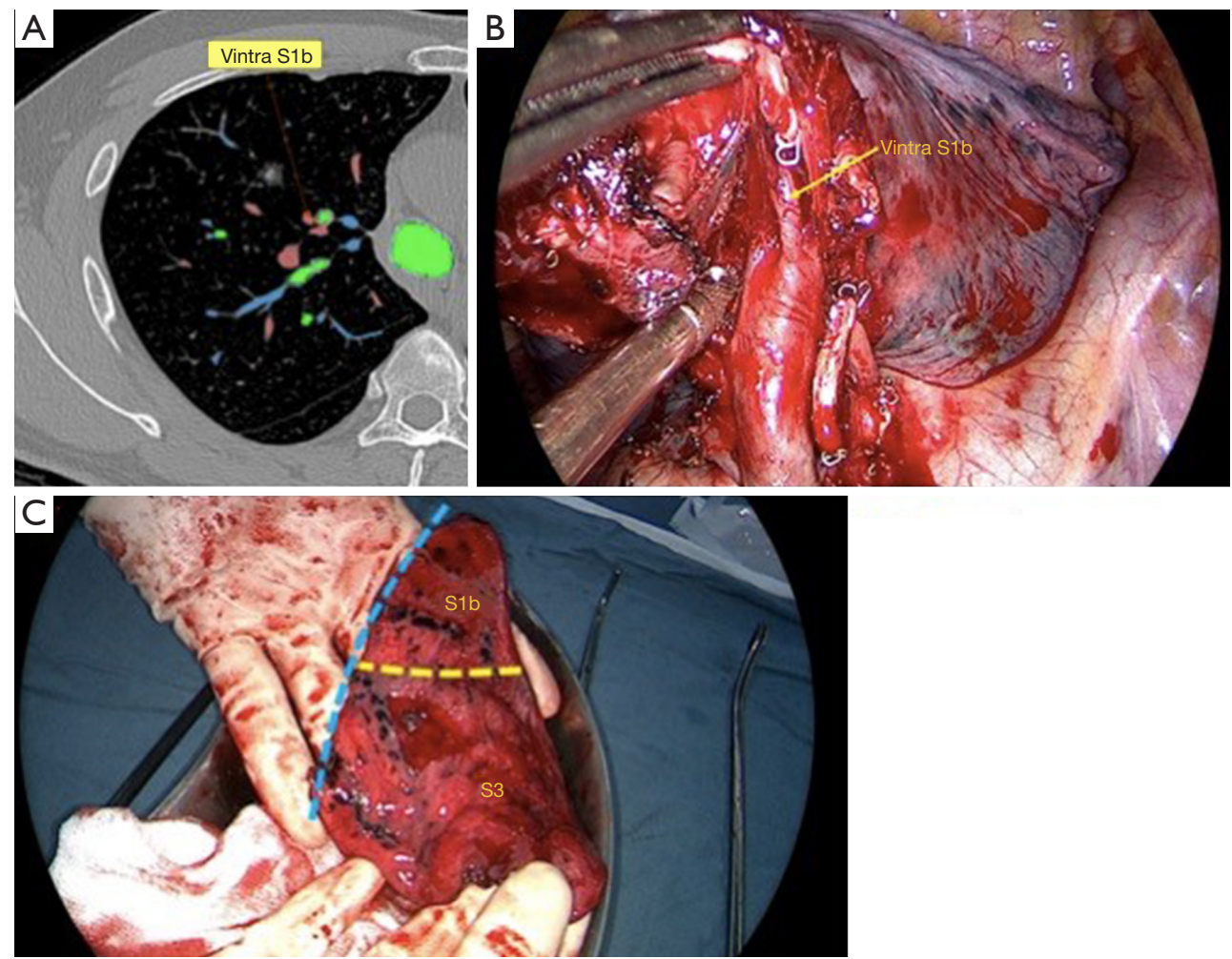

Figure 5 Comparison of CT finding and intraoperative findings. (A,B) Veins in S1b sub-segment; (C) the nodule adjacent to the V1b plane (after the lung tissue was removed).

As surgeons become more familiar with the anatomy of the lung segments, precise identification of the anatomical structures of lung segment by preoperative thin-slice 2D CT is gradually turning into reality.

The right upper lobe includes three segments: apical (S1), posterior (S2), and anterior (S3). The bronchus, as the core structure of the segment, need to be identified first. The distributing of the bronchus can be traced via the continuous thin-slice 2D CT to differentiate the lung segments where the bronchus is located. Most of the right upper lobes have three segmental bronchi: the apical bronchus (B1); runs upward and outward), the posterior bronchus (B2); runs backward and upward), and the anterior bronchus (B3); runs forward and downward). However, some patients including this reported case may have only two segmental bronchi, in which the apical sub-segment (B1a) originated from the posterior segment (B2), and the anterior sub-segment (B1b) originated from the anterior segment (B3). In general, the bronchus is relatively easy to identify on thin-slice 2D CT due to their hollow tubular structure.

After the identification of segmental bronchus, the second step is to identify the segmental veins, which is relatively challenging and requires good memory and spatial imagination. The right upper lobe veins include veins in the apical segment (V1a and V1b), the posterior segment (V2a, V2b, V2c, and V2t), and the anterior segment (V3a, V3b, and $V 3 c$ ). These veins distribute between the bronchus, and should be carefully traced until they merged into the atrium: V1b is located between the apical and anterior segments; V2a is located between the apical and posterior segments; and V2c is located between the posterior and anterior segments. According to the continuous dynamic reading of thin-slice CT images, each structure represents a different point on the plane; in addition, good spatial imagination helps with learning the distributing of the veins in the lung segments and the planes between segments.

The last step is to identify the anatomy of the arteries. The arteries distribute along the bronchus, and they are closer to each other at the distal terminals. Therefore, the arteries can be memorized together with the bronchus. Notably, the root of the proximal artery is relatively far away from that of the bronchus; if it is difficult to identify 
the artery from its proximal portion, the artery needs to be identified from distal to proximal. Arteries in the apical segment (A1a, A1b) and arteries in the anterior segment (A3a and A3b) mostly originate from the apical anterior segmental artery, and some of the recurrent arteries (RecA2) also originate from the apical anterior segmental artery. They can be identified on CT by tracing the bronchus in the apical, anterior, and posterior segments. Another posterior ascending branch (AscA2) of the posterior segmental artery originates from the main pulmonary artery and accompanies the posterior bronchus; however, it may not exist in some patients. There may also be an AscA3a from the anterior segmental artery, which distributes along the bronchus in the anterior segment.

\section{Conclusions}

During thin-slice 2D CT scanning, the structures in the lung segments must be continuously identified slice by slice. Each structure should be continuously traced from the center to the periphery then from the periphery to the center. Simultaneously, 3D reconstruction results and intraoperative findings can be used to train spatial imagination to speed up 2D identification. Bronchus is the core structure in the lung segments and needs to be clearly identified first. The names of the bronchi should be memorized because they represent the distributing directions of the bronchus and can facilitate their accurate identification on 2D CT. The veins run between the bronchus and should be carefully identified from distal to proximal until they merge into the atrium. The arteries accompany the bronchus. They should be carefully identified from distal to proximal from the sub-segment to the arterial root; through this, the posterior ascending artery, posterior recurrent artery, anterior ascending artery, and anterior recurrent artery can be well recognized.

\section{Acknowledgments}

Funding: None.

\section{Footnote}

Conflicts of Interest: All authors have completed the ICMJE uniform disclosure form (available at http://dx.doi. org/10.21037/jtd.2020.03.56). JS serves as an unpaid Section Editor of Fournal of Thoracic Disease from Jan 2020 to Dec 2020. The other authors have no conflicts of interest to declare.

Ethical Statement: The authors are accountable for all aspects of the work in ensuring that questions related to the accuracy or integrity of any part of the work are appropriately investigated and resolved. Written informed consent was obtained from the patient for publication of this study and any accompanying images. A copy of the written consent is available for review by the Editor-inChief of this journal.

Open Access Statement: This is an Open Access article distributed in accordance with the Creative Commons Attribution-NonCommercial-NoDerivs 4.0 International License (CC BY-NC-ND 4.0), which permits the noncommercial replication and distribution of the article with the strict proviso that no changes or edits are made and the original work is properly cited (including links to both the formal publication through the relevant DOI and the license). See: https://creativecommons.org/licenses/by-nc-nd/4.0/.

\section{References}

1. Siegel RL, Miller KD, Jemal A. Cancer statistics, 2019. CA Cancer J Clin 2019;69:7-34.

2. Aberle DR, DeMello S, Berg CD, et al. Results of the two incidence screenings in the National Lung Screening Trial. N Engl J Med 2013;369:920-31.

3. Ginsberg RJ, Rubinstein LV. Randomized trial of lobectomy versus limited resection for T1 N0 non-small cell lung cancer. Lung Cancer Study Group. Ann Thorac Surg 1995;60:615-22; discussion 622-3.

4. Dai C, Shen J, Ren Y, et al. Choice of Surgical Procedure for Patients With Non-Small-Cell Lung Cancer $\leq 1 \mathrm{~cm}$ or $>1$ to $2 \mathrm{~cm}$ Among Lobectomy, Segmentectomy, and Wedge Resection: A Population-Based Study. J Clin Oncol 2016;34:3175-82.

5. Suzuki K, Saji H, Aokage K, et al. Comparison of pulmonary segmentectomy and lobectomy: Safety results of a randomized trial. J Thorac Cardiovasc Surg 2019;158:895-907.

Cite this article as: Wang R, Zhang Y, Hu Q, Jin K, Huang G, Shen J, Bao F. Identification of the segmental structures of the right upper lobe of the lung using non-enhanced thin-slice CT. J Thorac Dis 2020;12(4):1639-1644. doi: 10.21037/jtd.2020.03.56 